\title{
Gastrointestinal disease in Sjogren's syndrome: related to food hypersensitivities
}

\author{
Christine Kim-Lee ${ }^{1}$, Lakshmanan Suresh ${ }^{2,3}$ and Julian L. Ambrus Jr. ${ }^{1 *}$ (D)
}

\begin{abstract}
Patients with Sjogren's syndrome (SS) frequently have irritable bowel like symptoms (IBS). Some have celiac sprue. The current studies were designed to examine the presence of food hypersensitivities in a population of patients with SS and IBS. Ten patients were selected from the autoimmune disease clinics at SUNY at Buffalo who had SS and IBS symptoms. Food hypersensitivities were determined by specific $\operatorname{lgG}$ ImmunoCAP ${ }^{\circledR}$ assays. Symptoms of abdominal pain, bloating, diarrhea and joint pain were eliminated with dietary restriction of foods to which hypersensitivity was demonstrated. Symptoms recurred with re-institution of offending foods. Resolution of fatigue required elimination of offending foods as well as treatment of underlying metabolic disorders. The presence of IBS in patients with SS should lead to investigation of food hypersensitivities as possible culprits.
\end{abstract}

\section{Background}

Sjogren's syndrome (SS) is a common autoimmune disease characterized by destruction of the salivary and lacrimal glands leading to symptoms of dry eyes and dry mouth. Extra-glandular manifestations commonly include lung disease, kidney disease and lymphoma (Delaleu et al. 2005; Mavragani and Moutsopoulos 2014). Lymphomas may occur in the salivary glands but also frequently in the gastrointestinal tract (Liang et al. 2014; Mariette 1999). Gastrointestinal disease in SS may occur in one quarter of the patients and include dysphagia, gastritis, motility disorders, pancreatitis, pancreatic insufficiency, pernicious anemia, autoimmune hepatitis and symptoms consistent with irritable bowel syndrome (IBS), abdominal pain, diarrhea, constipation, bloating, flatulence, vomiting and nausea (Ebert 2012; Bengtsson et al. 2011). Some patients with SS and IBS are found to have celiac sprue (Conti et al. 2015). The current studies were undertaken to evaluate if patients with SS and IBS symptoms in our clinic population had hypersensitivities to particular foods, whether or not they met criteria

\footnotetext{
*Correspondence: jambrus@buffalo.edu

1 Department of Medicine, SUNY at Buffalo School of Medicine,

Buffalo 14203, NY, USA

Full list of author information is available at the end of the article
}

for celiac sprue, and if elimination of the offending foods would alleviate their symptoms.

\section{Methods}

Ten patients were identified from the adult autoimmune disease clinics at SUNY at Buffalo School of Medicine who met the 2012 ACR criteria for the diagnosis of SS and complained of abdominal pain, bloating, diarrhea and fatigue associated with the ingestions of particular foods. All had been diagnosed with IBS or celiac sprue by gastroenterologists. In some cases, patients had identified particular foods as culprits, but in most cases not. The presence of fatigue and joint pain were determined by questioning the patients.

Determination of food hypersensitivities was performed using the ImmunoCAP ${ }^{\circledR}$ system (Phadia $A B$, Uppsala, Sweden) to measure food-specific IgG. The food was immobilized on the solid phase and IgG antibodies were detected with a fluorescently labeled antibody. The latter detected all subclasses of IgG. The IgG scoring system was established by the manufacturer (Viracor-IBT Laboratories, Lee's Summit, MO) with a standard curve used to calculate the specific IgG concentrations. The calibrators were referenced to the International reference preparation for serum Ig. The assay range has a lower limit of quantitation of $2 \mu \mathrm{g} / \mathrm{mL}$ and an upper limit of

\section{Springer}


quantitation of $200 \mu \mathrm{g} / \mathrm{mL}$ for specific IgG. The assay was reported as positive when food-specific IgG levels were higher than $2 \mathrm{mcg} / \mathrm{mL}$ (Atkinson et al. 2004).

In each case, patients met with a dietician and developed a diet eliminating the foods identified by these assays. At the end of a 6 months period, patients attempted to reinstitute foods, one at a time. Symptoms were recorded before and during the elimination diet as well as with the reinstitution of the culprit foods. These studies were approved by the IRB, SUNY at Buffalo School of Medicine.

\section{Results}

The characteristics of the ten SS patients studied are shown in Table 1. This group consisted of 8 females and 2 males, age 28-63 years (mean 48.2 years). Most patients had SS for 2 years or less, although one patient had disease for 3 years, two for 5 years and one for 18 years. The symptoms of bloating, diarrhea, joint pain and fatigue often preceded the diagnosis of SS. Interestingly, although perhaps because of the nature of the autoimmune disease clinics at SUNY at Buffalo School of Medicine that have a large number of patients with metabolic muscle diseases, all of the patients were identified to have metabolic myopathies. Five patients had carnitine palmitoyl transferase deficiency. One patient had complex 2 disorder of the mitochondrial respiratory chain, and another had lactate dehydrogenase deficiency. All of these patient had their diagnosis confirmed based on muscle biopsies. Three additional patients had their mitochondrial dysfunction demonstrated by elevated lactic acid at rest on several occasions, without an alternative explanation. Besides SS and metabolic disorders, two patients had psoriasis and three had anti-phospholipid antibody syndrome.
The clinical features that brought these patients to medical attention were abdominal pain, bloating, diarrhea and fatigue associated in many cases with joint pains involving predominantly the wrists, knees and ankles. Gastroenterologist had performed endoscopies and colonoscopies on all of these patients. One patients was diagnosed with celiac sprue, but still had ongoing symptoms after elimination of gluten. The remainder of the patients had been given the diagnosis of IBS. All of the patients had deficiencies in 25-OH Vitamin D, often with levels less than $20 \mathrm{ng} / \mathrm{ml}$ (Normal 30-100 ng/ml), suggesting some degree of malabsorption (Table 2). One patient had an episode of uveitis that was unexplained, but may have be related to gastrointestinal inflammation (Rachitskaya et al. 2010).

The ImmunoCAP ${ }^{\circledR}$ testing for IgG reactivity to foods revealed reactivities to multiple food in all cases (Table 2). Reactivity to wheat and dairy was present in all but one patient. Other common reactivities were to eggs, beef and corn. All of the patients had autoantibodies associated with SS, ANA, RF, anti-Ro, anti-salivary gland protein 1 (SP1), anti-carbonic anhydrase 6 (CA6) and/or anti-parotid secretory protein (Kyriakidis et al. 2014; Shen et al. 2012, 2014). Most of the patients had antibodies associated with inflammation in the gastrointestinal tract, five patients had anti-S. cerevesiae antibodies (ASCA), one patient had atypical pANCA and one patient had anti-gliadin antibodies (Torok et al. 2004; Beniwal and Harrell 2010).

As shown in Table 3, all patients, except for two, were able to eliminate all the foods from their diets to which they had hypersensitivity. The eight patients with complete elimination diets had full resolution of abdominal pain, bloating, diarrhea and joint pain on the restricted diet. The two patients with incomplete elimination diets

Table 1 The Table summarizes demographic data in ten patients studied with SS and IBS-like symptoms

\begin{tabular}{lllll}
\hline Patient & $\begin{array}{l}\text { Age } \\
\text { (years) }\end{array}$ & Sex & $\begin{array}{l}\text { Duration Sjogren's } \\
\text { syndrome (years) }\end{array}$ & Other medical comorbidities \\
\hline 1 & 30 & F & 1 & Psoriasis; mitochondrial dysfunction \\
2 & 28 & F & 2 & Carnitine palmitoyl transferase deficiency \\
3 & 39 & F & 2 & Polymyositis; carnitine palmitoyl transferase deficiency \\
4 & 50 & M & 2 & Carnitine palmitoyl transferase deficiency \\
5 & 53 & M & 5 & Psoriasis; APLA; mitochondrial dysfunction \\
6 & 56 & F & 1 & Complex 2 disorder mitochondrial respiratory chain; APLA; celiac sprue \\
7 & 54 & F & 2 & Lactate dehydrogenase deficiency \\
8 & 49 & F & 5 & Carnitine palmitoyl transferase deficiency \\
9 & 60 & F & 3 & Carnitine palmitoyl transferase deficiency; APLA \\
10 & 63 & F & 18 & Mitochondrial dysfunction
\end{tabular}


Table 2 This table summarizes the foods to which the patients had positive food reactivities based on specific IgG ImmunoCAP ${ }^{\circledR}$ studies

\begin{tabular}{lll}
\hline Patient & Food hypersensitivities & Autoantibodies \\
\hline 1 & Wheat, dairy, eggs, beef, corn & ASCA, ANA \\
2 & Wheat, dairy, beef, corn, rice, tomato, carrot, broccoli, apple & ASCA, ANA, anti-SP1 \\
3 & Wheat, dairy & ASCA, ANA \\
4 & Wheat, eggs, dairy, banana, beef, rice, corn, almond & Atypical pANCA, anti-CA6, anti-PSP \\
5 & Wheat, soy, dairy, corn banana, egg, peanut, shrimp, almond, tomato & ANA, RF \\
7 & Rice, almonds, eggs, banana & ASCA, ANA, anti-SP1 \\
9 & Wheat, dairy, tomato, eggs & Anti-Ro \\
10 & Wheat, eggs, dairy, soy, corn, strawberry & ASCA, ANA, anti-Ro \\
\hline
\end{tabular}

Autoantibodies were determined by ELISA assays

ASCA anti-Saccharomyces cerevesiae antibodies, anti-CA6 anti-carbonic anhydrase 6, anti-PSP anti-parotid secretory protein, anti-SP1 anti-salivary gland protein 1

Table 3 This table summarizes the symptoms attributable to food hypersensitivities in each of the patients, their symptoms on their elimination diets and the foods added back that caused the initial symptoms to recur

\begin{tabular}{|c|c|c|c|}
\hline Patient & $\begin{array}{l}\text { Symptoms ascribed to food hypersensi- } \\
\text { tivity }\end{array}$ & Symptoms remaining on food elimination & $\begin{array}{l}\text { Recurrence of all symptoms with food } \\
\text { re-challenge }\end{array}$ \\
\hline 1 & Bloating, diarrhea, joint pain, fatigue & Mild fatigue & Yes; wheat or dairy \\
\hline 2 & Bloating, diarrhea, joint pain, fatigue & $\begin{array}{l}\text { All improved, but never complete food } \\
\text { elimination }\end{array}$ & Yes; wheat, corn or dairy \\
\hline 3 & Bloating, diarrhea, joint pain, fatigue & Mild fatigue & Yes; wheat or dairy \\
\hline 4 & Bloating, diarrhea, joint pain, fatigue, scleritis & Mild fatigue & Yes; wheat, eggs, dairy or beef \\
\hline 5 & Bloating, joint pain, fatigue & Mild fatigue & Yes; wheat or dairy \\
\hline 6 & Bloating, diarrhea, joint pain, fatigue & Mild fatigue & $\begin{array}{l}\text { Yes; dairy, corn, soy or rice; she would not try } \\
\text { wheat }\end{array}$ \\
\hline 7 & Bloating, diarrhea, joint pain, fatigue & Fatigue & Yes; rice, eggs or banana \\
\hline 8 & Bloating, diarrhea, joint pain, fatigue & Mild fatigue & Yes; wheat or diary or eggs \\
\hline 9 & Bloating, diarrhea, joint pain, fatigue & $\begin{array}{l}\text { All improved, but never complete food } \\
\text { elimination }\end{array}$ & Yes; wheat or dairy \\
\hline 10 & Bloating, diarrhea, joint pain, fatigue & Mild fatigue & Yes; wheat, corn or dairy \\
\hline
\end{tabular}

did eliminate wheat and dairy from their diets and noted improvement but not resolution of their abdominal symptoms. In all cases, symptoms returned when culprit foods were re-introduced. Re-introduction of food was done one food at a time. In most cases, ingestion of either wheat or dairy returned the gastrointestinal symptoms that had occurred before the elimination diet. Over time, some patients were able to re-introduce some foods without symptoms, such as tomato, pork and rice.

While fatigue was a major component in the symptoms of all these patients, elimination diets improved but did not resolve it. Further improvement in fatigue came from addressing the underlying metabolic diseases. Patients with mitochondrial dysfunction from any cause received CoQ10, creatine, carnitine, folic acid and alpha-lipoic acid (Tarnopolsky 2008; Abdullah et al. 2012; Ambrus
2009). The patient with lactate dehydrogenase deficiency was treated with a diet avoiding complex carbohydrates and rich in simple sugars (Weinstein and Wolfsdorf 2002).

\section{Discussion}

The current study was an observational study made on a group of patients with SS derived from a University autoimmune disease clinic that also specializes in metabolic muscle diseases. It is therefore a very selected group of patients. Nonetheless, these patients presented with symptoms that are common to SS patients everywhere and often ascribed to IBS, bloating, diarrhea, joint pain and fatigue (Bengtsson et al. 2011; Daniels et al. 2011). Patients were identified to have various food hypersensitivities by IgG reactivity to these foods. Diets eliminating 
these foods led to resolution of symptoms that recurred with re-introduction of the foods. Resolution of fatigue required attention to not only the food hypersensitivities but also to metabolic disorders.

The incidence of celiac sprue, or gluten hypersensitivity, is estimated to be $1 \%$ of the population and to be present in many patients with Sjogren's syndrome (Rashtak et al. 2009). Celiac sprue is associated with abdominal pain, diarrhea, bloating, arthritis, uveitis, fatigue, iron deficiency and various vitamin deficiencies, including vitamin D (McGough and Cummings 2005). It is known that many patients with celiac sprue to not respond to elimination of gluten alone. Many theories exist regarding the failure of gluten elimination alone to resolve symptoms in these patients (Farrell and Kelly 2002).

More recently, it has been recognized that hypersensitivity reactions can occur to food other than gluten. In fact, gastroenterologists discuss the entity of non-celiac sprue wheat hypersensitivity as a common entity (Battais et al. 2003). The idea that hypersensitivities to other foods, such as dairy, eggs and beef, giving similar symptoms to celiac sprue is a relatively new concept that is undergoing intense study (Zuo et al. 2007). Allergist studying "food allergies" recognize IgE mediated reactions to foods that cause immediate diarrhea, skin rash and swelling upon ingestion of the foods (Kurowski and Boxer 2008). It is being recognized more by gastroenterologists than allergists that hypersensitivities to foods cause delayed reactions (8-48 $\mathrm{h}$ after ingestion) that most commonly include bloating, diarrhea and fatigue (Jyonouchi 2008; Wolfe and Aceves 2011). It is also recognized that any type of gastrointestinal inflammation can lead to secondary arthralgia/arthritis most commonly involving the wrists, knees and ankles (Holden et al. 2003). Our patients clearly had symptoms suggestive of food hypersensitivities, with blood test demonstrating food sensitivities and resolution of symptoms with elimination diets that recurred with reintroduction of foods. These data suggest that hypersensitivity to these foods was a critical driver for these symptoms. The mechanism by which these food hypersensitivities caused these symptoms is entirely unclear, and should be the topic of future studies.

That these symptoms occurred in a population of patients with SS raises several interesting issues. First, it could be that food hypersensitivities and SS are both common entities and we happened to find patients in which they co-exist. This would be supported by the fact that many of the patients had gastrointestinal symptoms before the identification of SS. However, SS can frequently exist for several years before it is diagnosed (Akpek et al. 2015). Second, the SS patients may have been a unique subset. It is true that all the patients had autoantibodies and met ACR criteria for SS, however, many lacked anti-Ro or anti-La antibodies. Several patients had anti-SP1 antibodies. Whether anti-SP1, anti-CA6 and/or anti-PSP denote different subsets of SS is currently being evaluated in several studies (Suresh et al. 2015; Ambrus et al. 2012). Lastly, all of the patients in these groups of SS patients had underlying metabolic diseases. It is possible that the metabolic diseases participated in the development of both the food hypersensitivities and the SS. One scenario that could be envisioned, and would have to be evaluated in future studies, is that the underlying metabolic disease led to increased susceptibility to infection and increased difficulty in clearing infections. Infections in the gastrointestinal tract, with enteroviruses for example, would lead to reactivity to foods that were commonly in the diet. At the same time, infections in the salivary and lacrimal glands, with CMV or mumps for example, could initiate the events leading to SS. Many other scenarios can be envisioned. The bias of this study is that patients were selected from a clinic that specializes in autoimmune diseases and metabolic diseases. Further studies will have to evaluate these issues in a broader population of SS patients.

In summary, patients selected from an academic autoimmune disease and metabolic disease clinic identified 10 patients with SS and IBS like symptoms that were caused by hypersensitivity to foods, the most common being wheat and dairy. Fatigue in these patients was related to food hypersensitivities and underlying metabolic disorders. Treatment of all of these issues is necessary to help these patients feel better and have a better quality of life.

\section{Abbreviations \\ SS: Sjogren's syndrome; IBS: irritable bowel syndrome; ASCA: anti-Saccharo- myces cerevesiae antibodies; Anti-CA6: anti-carbonic anhydrase 6; Anti-PSP: anti-parotid secretory protein; Anti-SP1: anti-salivary gland protein 1; Atypical pANCA: atypical peri-nuclear anti-neutrophil cytoplasmic antibodies; APLA: anti-phospholipid antibody.}

\section{Authors' contributions}

$\mathrm{CKL}$ helped with the care of the patients, the design of the study and the writing of the manuscript. She managed the distribution and collection of the questionnaires. LS ran the autoantibody assays and participated in the writing of the manuscript. JLA cared for the patients, designed the study and helped write the manuscript. All authors read and approved the final manuscript.

\section{Author details}

${ }^{1}$ Department of Medicine, SUNY at Buffalo School of Medicine, Buffalo 14203, NY, USA. ${ }^{2}$ Department of Oral Diagnostic Sciences, SUNY at Buffalo School of Dental Medicine, Buffalo 14214, NY, USA. ${ }^{3}$ Immco Diagnostics, 640 Ellicott Street, Buffalo 14203, NY, USA.

\section{Acknowledgements}

The authors would like to thank the patients and nurses who participated in the successful completion of these studies.

\section{Competing interests}

The authors declare that they have no competing interests.

Received: 19 November 2015 Accepted: 24 November 2015 Published online: 12 December 2015 


\section{References}

Abdullah M, Vishwanath S, Elbalkhi A, Ambrus JL Jr (2012) Mitochondrial myopathy presenting as fibromyalgia: a case report. J Med Case Rep 6(1):55

Akpek EK, Mathews P, Hahn S, Hessen M, Kim J, Grader-BeckT et al (2015) Ocular and systemic morbidity in a longitudinal cohort of sjogren's syndrome. Ophthalmology 122(1):56-61

Ambrus JJ (2009) Experience With Mitochondrial Dysfunction In A Population of adult patients seen in an academic adult rheumatology and immunology practice over a period of eight years. J Med 37:1-12

Ambrus JJ, Shen L, Lindemann P, Kowal M, Suresh L (2012) Changes in autoantibody production during different stages of Sjogren's disease. 8th Intern Congr Autoimmun 8:474

Atkinson W, Sheldon TA, Shaath N, Whorwell PJ (2004) Food elimination based on IgG antibodies in irritable bowel syndrome: a randomised controlled trial. Gut 53(10):1459-1464

Battais F, Pineau F, Popineau Y, Aparicio C, Kanny G, Guerin L et al (2003) Food allergy to wheat: identification of immunogloglin $E$ and immunoglobulin G-binding proteins with sequential extracts and purified proteins from wheat flour. Clin Exp Allergy 33(7):962-970

Bengtsson M, Hammar O, Mandl T, Ohlsson B (2011) Evaluation of gastrointestinal symptoms in different patient groups using the visual analogue scale for irritable bowel syndrome (VAS-IBS). BMC Gastroenterol 11:122

Beniwal P, Harrell L (2010) The status of diagnostic markers for inflammatory bowel disease. Curr Gastroenterol Rep 12(6):479-484

Conti V, Leone MC, Casato M, Nicoli M, Granata G, Carlesimo M (2015) High prevalence of gluten sensitivity in a cohort of patients with undifferentiated connective tissue disease. Eur Ann Allergy Clin Immunol 47(2):54-57

Daniels TE, Cox D, Shiboski CH, Schiodt M, Wu A, Lanfranchi H et al (2011) Associations between salivary gland histopathologic diagnoses and phenotypic features of sjogren's syndrome among 1,726 registry participants. Arthritis Rheum 63(7):2021-2030

Delaleu N, Jonsson R, Koller MM (2005) Sjogren's syndrome. Eur J Oral Sci 113(2):101-113

Ebert EC (2012) Gastrointestinal and hepatic manifestations of Sjogren syndrome. J Clin Gastroenterol 46(1):25-30

Farrell RJ, Kelly CP (2002) Current concepts: celiac sprue. N Engl J Med 346(3):180-188

Holden W, Orchard T, Wordsworth P (2003) Enteropathic arthritis. Rheum Dis Clin N Am 29(3):513-530

Jyonouchi H (2008) Non-IgE mediated food allergy. Inflamm Allergy Drug Targets 7(3):173-180

Kurowski K, Boxer RW (2008) Food allergies: detection and management. Am Fam Physician 77(12):1678-1686
Kyriakidis NC, Kapsogeorgou EK, Tzioufas AG (2014) A comprehensive review of autoantibodies in primary Sjogren's syndrome: clinical phenotypes and regulatory mechanisms. J Autoimmun 51C:67-74

Liang Y, Yang ZX, Qin BD, Zhong RQ (2014) Primary Sjogren's syndrome and malignancy risk: a systematic review and meta-analysis. Ann Rheum Dis 73(6):1151-1156

Mariette X (1999) Lymphomas in patients with Sjogren's syndrome: review of the literature and physiopathologic hypothesis. Leuk Lymphoma 33(1-2):93-99

Mavragani CP, Moutsopoulos HM (2014) Sjogren's syndrome. Annu Rev Pathol 9(9):273-285

McGough N, Cummings JH (2005) Coeliac disease: a diverse clinical syndrome caused by intolerance of wheat, barley and rye. Proc Nutr Soc 64(4):434-450

Rachitskaya A, Mandelcorn ED, Albini TA (2010) An update on the cause and treatment of scleritis. Curr Opin Ophthalmol 21(6):463-467

Rashtak S, Marietta EV, Murray JA (2009) Celiac sprue: a unique autoimmune disorder. Expert Rev Clin Immunol 5(5):593-604

Shen L, Suresh L, Lindemann M, Xuan J, Kowal P, Malyavantham K et al (2012) Novel autoantibodies in Sjogren's syndrome. Clin Immunol 145(3):251-255

Shen L, Kapsogeorgou EK, Yu MX, Suresh L, Malyavantham K, Tzioufas AG et a (2014) Evaluation of salivary gland protein 1 antibodies in patients with primary and secondary Sjogren's syndrome. Clin Immunol 155(1):42-46

Suresh L, Malyavantham K, Shen L, Ambrus JL Jr (2015) Investigation of novel autoantibodies in Sjogren's syndrome utilizing Sera from the Sjogren's international collaborative clinical alliance cohort. BMC Ophthalmol 15(1):38

Tarnopolsky MA (2008) The mitochondrial cocktail: rationale for combined nutraceutical therapy in mitochondrial cytopathies. Adv Drug Deliv Rev 60(13-14):1561-1567

Torok HP, Glas J, Gruber R, Brumberger V, Strasser C, Kellner H et al (2004) Inflammatory bowel disease-specific autoantibodies in HLA-B27-associated spondyloarthropathies: increased prevalence of ASCA and pANCA. Digestion 70(1):49-54

Weinstein DA, Wolfsdorf JI (2002) Glycogen storage diseases: a primer for clinicians. Endocrinologist 12(6):531-538

Wolfe JL, Aceves SS (2011) Gastrointestinal manifestations of food allergies. Pediatr Clin N Am 58(2):389-405

Zuo XL, Li YQ, Li WJ, Guo YT, Lu XF, Li JM et al (2007) Alterations of food antigen-specific serum immunoglobulins $\mathrm{G}$ and $\mathrm{E}$ antibodies in patients with irritable bowel syndrome and functional dyspepsia. Clin Exp Allergy 37(6):823-830

\section{Submit your manuscript to a SpringerOpen ${ }^{\odot}$ journal and benefit from:}

- Convenient online submission

- Rigorous peer review

- Immediate publication on acceptance

- Open access: articles freely available online

- High visibility within the field

- Retaining the copyright to your article

Submit your next manuscript at $\boldsymbol{~ s p r i n g e r o p e n . c o m ~}$ 